\title{
Cancer protective mutations: looking for the needle in the haystack
}

Even though modern laboratory techniques have represented a giant step in our understanding of genetics, which is probably best symbolized by $t$

$h$

recent completion of the sequencing of the human genome, only an adequate interpretation of this information will make that vast amount of knowledge useful. In the field of Oncology, a large number of genetic alterations which increase the risk of cancer have been described. An important number of them have been detected through the study of individuals either affected by multiple tumor syndromes, and/or with a known increased familial risk of developing cancer. Some well known examples are p53 germ line mutations in patients with the Li-Fraumeni syndrome, inactivation of both copies of the retinoblastoma tumor-suppressor gene in patients with hereditary retinoblastoma, ${ }^{2,3}$ as was wisely predicted by Knudson- ${ }^{4}$ or mutations in BRCA-1, a gene which was identified in families with earlyonset breast cancer ${ }^{5}$. It is, however, surprising that the opposite strategy has not been pursued: to search for potential protective genetic alterations or host factors in patients with an unusually low rate of developing cancer. This issue has probably not been carefully evaluated in oncology, despite the fact that it has been described in other diseases. Two interesting examples can be used to illustrate successful strategies to identify protective

mutations. Possibly the most interesting yet difficult one, is to study the genotype of subjects with an unique, very characteristic phenotype. Perhaps the most outstanding example is the identification of a deletion in the gene encoding the chemokine coreceptor CCR-5, which confers to homozygotic individuals complete protection against infection by certain strains of the human

Rev Oncología 2001; 4: 169-171

The opinions expressed in this article do not necessarily reflect the opinions of the institutions with which the authors are affiliated. inmunodeficiency virus (HIV) ${ }^{6,7}$. At least one other heterozygotic CCR-5 mutation, when associated with the mentioned deletion in the other allele, has been shown to produce the same effect ${ }^{8}$. Since the CCR-5 mutations had not been associated with any abnormalities, an astute observation of the fact that some individuals highly exposed to HIV never developed the infection was required to identify them ${ }^{9}$. Secondary to this observation, CCR-5 has become a relevant target in the investigation of HIV infection. A second strategy for identifying protective mutations is to study the expression of gene polymorphisms which are supposed to be related with the incidence of a disease in cases and controls studies. A recent example is the relation of certain factor VII genotypes with the risk of myocardial infarction ${ }^{10}$. High plasma levels of coagulation factor VII have been suggested to be related to risk of death due to coronary artery disease, and polymorphisms in the factor VII gene are associated with variations in levels of factor VII. Therefore, such polymorphisms were studied in 311 individuals with severe, angiographically documented coronary atherosclerosis, of whom 175 had a history of previous myocardial infarction, and the rest did not. Among patients with no history of previous myocardial infarction, there was a significantly superior number of patients with determined genotypes, than among patients with myocardial infarction, wich suggests a protective action for such genotypes.

These

types of studies must be interpreted with a critical mind, because they are subject to a potentially high risk of bias, as reviewed by Gambaro et $\mathrm{al}^{11}$. Nonetheless, this example is particularly interesting, since the controls were asymptomatic subjects with no history of myocardial infarction despite having a strong risk factor for such an event (documented coronary atherosclerosis), rather than being just normal healthy subjects (although the study did include an additio-nal control group composed of healthy subjects). Therefore, there was a greater chance of finding true 
protective factors, rather than just absence of disease, as could be expected from a control group formed by individuals with a normal population risk. The bottom line of both examples is that through the study of subjects with a lower than normal risk of developing a disease, protective host factors for that disease might be identified, just as the study of subjects with increased risk of developing cancer leads to the discovery of cancer related genetic alterations.

Since the risk of developing cancer is not uniform, it is possible to hypothesize that, just as some individuals present an increased risk, other subjects may have lower risk than would be expected for their environment and habits. These subjects would represent the left tail of a Gaussian distribution showing the risk of developing cancer. If so, the identification and the study of these subjects could disclose genetic alterations or other intrinsic host factors, such as a special immune response, conferring a partial, or perhaps even a total protection against certain neoplastic disorders. The detection of these alterations would not only increase our knowledge of cancer but could also yield potentially useful targets against it. Furthermore, we must observe that cancer is a frequent and ancient disease (probably, inherent to life itself), and it is therefore possible that such mutations may have developed during evolution. The search for relevant protective factors is especially appealing in the field of oncology, because once a tumor develops, rather than expressing one single abnormality, it seems to undergo a large number of further genetic alterations which will ultimately make it resistant to any kind of treatment. Therefore, the identification of factors which could avoid the development of the tumor in the first place seems particularly interesting.

A considerable number of polymorphisms which supposedly confer some degree of protection against cancer have been identified. Some polymorphisms in the methylenetetrahydrofolate reductase gene have been associated with a decreased risk of developing acute lymphocytic leukemia $^{12}$ or colorectal cancer, ${ }^{13,14}$ in certain population subsets. Polymorphisms of enzymes like microsomal epoxide hydrolase ${ }^{15}$, myeloperoxidase ${ }^{16-19}$ or $\mathrm{NAD}(\mathrm{P}) \mathrm{H}$ : quinone reducta$\mathrm{se}^{20}$, which are involved in the metabolism of determined carcinogens have also been related to some degree of protection against developing lung cancer or colorectal cancer in some population subsets and/or ethnic groups, even though other studies, such as that of microsomal epoxide hydrolase, have shown no association ${ }^{22}$, or even an inverse relationship ${ }^{23}$. Determined genotypes of some cytochrome P450 enzymes, such as CYP1A1 or CYP2D6, have also been correlated with a decreased risk of lung cancer, although meta-analyses have failed to confirm this observation $^{24,25}$ or have just described a small protective effect with a nonappreciable relationship to individual susceptibility ${ }^{26}$. Two common polymorphisms of the p21WAF1/Cipl gene have been correlated with a potential protective role against ovarian cancer ${ }^{27}$. Certain genotypes of the glutathione S-transferase M1 have also been related with the risk of lung cancer and other aerodigestive tract cancers, but again a meta-analyses has failed to confirm such results ${ }^{28}$. Finally, some HLA alleles have been linked in cases and controls studies with a decreased susceptibility to renal cell carcinoma ${ }^{29}$, melanoma ${ }^{30}$ or lung cancer ${ }^{31}$, and even homozygotic women for determined polymorphic alleles of the BRCA-1 gene, have been associated with a decreased risk of breast cancer ${ }^{32}$.

The ambiguous and clinically not very relevant results of these studies may be explained by methodological flaws, as detailed elsewhere ${ }^{11}$, but may also be related to the poor selection of the study popu-lations. In contrast with the studies previously mentioned, in which the individuals were selected by a very characteristic phenotype (a definite protection against developing HIV or myocardial infarction) the latter studies used as control groups normal subjects. In these subjects, the risk of developing cancer was probably neither increased, nor decreased (with perhaps the only exception being that some groups were formed by smokers)

and it was therefore unlikely that clinically rele$\mathrm{v} \quad \mathrm{a}$ n t information would be discovered. Instead, the approach should be to study subjects with a markedly reduced familial or individual risk of developing cancer.

Families with a very low or ideally null incidence of cancer over several generations, perhaps despite crossing with high risk families, could be said to have a reduced familiar risk. Subjects that do not develop cancer despite important exposure to well-known extrinsic risk factors (such as heavy exposure to radiation) or intrinsic ones (such as a potential subject with familiar adenomatous polyposis developing cancer significantly later than would be expected or not developing it at all) would have a reduced individual risk. A different, yet perhaps valid approach, could be to study subjects who are unexplained long-term survivors of neoplasms which are supposed to be incurable, such as advanced non-small cell lung cancer. Combina- 
tions of these strategies or different ones could also be pursued, realizing that the chance of yielding positive results will be directly related to the discrepancy between the risk of developing cancer and the actual phenotype. If such families or individuals are found, it would be naive to attribute their particular phenotype to chance, at least until other causes have been ruled out.

In summary, while cancer research is mainly focused on the investigation of cancer itself (through the study of neoplastic cells, or of individuals with cancer or with an increased risk of developing the disease) another valid strategy could be to try to identify subjects with a lower than expected incidence of cancer which could be attributed to intrinsic factors, and to study the causes of this decreased incidence. This stra- tegy could provide a useful interpretation of the information that the sequencing of the human genome is yielding, and could help to increase our current knowledge of cancer and to discover new therapeutic strategies against this disease. Even though this strategy can be as difficult to perform as finding a needle in a haystack, such task is not impossible, if an adequate methodology is used, and as long as the needle is really there.

\section{References}

1. Malkin D, Li FP, Strong LC, et al. Germ line p53 mutations in a familial syndrome of breast cancer, sarcomas and other neoplasms. Science 1990; 250: 1.2331.238 .

2. Benedict WF, Murphree AL, Banerjee A, Spina CA, Sparkes MC, Sparkes RS. Patient with 13 chromosome deletion: evidence that the retinoblastoma gene is a recessive cancer gene. Science 1983; 219: 973-975.

3. Cavenee WK, Dryja TP, Phillips RA, et al. Expression of recessive alleles by chromosomal mechanisms in retinoblastoma. Nature 1983; 305: 779-784.

4. Knudson AG Jr. Mutation and cancer: statistical study of retinoblastoma. Proc Natl Acad Sci USA 1971; 68: 820-823.

5. Hall JM, Lee MK, Newman B, et al. Linkage of early-onset familial breast cancer to chromosome 17q21. Science $1990 ; 250$ : 1.684-1.689.

6. Liu R, Paxton WA, Choe S, et al. Homozygous defect in HIV-1 correceptor accounts for resistance of some multiple-exposed individuals to HIV-1 infection. Cell 1996; 86: 367-377.

7. Samson M, Libert F, Doranz BJ, et al. Resistance to HIV-1 infection in caucasian individuals bearing mutant alleles of the CCR-5 chemokine receptor gene. Nature 1996; 382: 722-725.

8. Quillent C, Oberlin E, Braun J, et al. HIV-1 resistance phenotype conferred by combination of two separate inherited mutations of CCR5 gene. Lancet 1998; 351: 14-18.
9. Rowland-Jones S, Sutton J, Ariyoshi K, et al. HIV-specific cytotoxic T-cells in HIV-exposed but uninfected Gambian women. Nat Med 1995; 1: 59-64.

10. Girelli D, Russo C, Ferraresi P, et al. Polymorphisms in the factor VII gene and the risk of myocardial infarction in patients with coronary artery disease. N Engl J Med 2000; 343: 774-780.

11. Gambaro G, Anglani F, D'Angelo A. Association studies of genetic polymorphisms and complex disease. Lancet 2000; 355: 308-311.

12. Skibola CF, Smith MT, Kane E, et al. Polymorphisms in the methylenetetrahydrofolate reductase gene are associated with susceptibility to acute leukemia in adults. Proc Natl Acad Sci USA 1999; 96: 12.81012.815 .

13. Chen J, Giovanucci E, Kelsey K, et al. A methylenetetrahydrofolate reductase polymorphism and the risk of colorectal cancer. Cancer Res 1996; 56: 4.8624.864 .

14. Ma J, Stampfer MJ, Giovanucci E, et al. Methylenetetrahydrofolate reductase polymorphism, dietary interactions, and risk of colorectal cancer. Cancer Res 1997; 57: 1.098-1.102.

15. London SJ, Smart J, Daly AK. Lung cancer risk in relation to genetic polymorphisms of microsomal epoxide hydrolase among African-Americans and Caucasians in Los Angeles County. Lung Cancer 2000; 28: 147155.

16. London SJ, Lehman TA, Taylor JA. Myeloperoxidase genetic polymorphism ad lung cancer risk. Cancer Res 1997; 57: 5.001-5.003.

17. Schabath MB, Spitz MR, Zhang X, Declos GL, Wu X. Genetic variants of myeloperoxidase and lung cancer risk. Carcinogenesis 2000; 21: 1.163-1.166.

18. Le Marchand L, Seifried A, Lum A, Wilkens LR. Association of the myeloperoxidase $-463 \mathrm{G} \rightarrow \mathrm{A}$ polymorphism with lung cancer risk. Cancer Epidemiol Biomarkers Prev 2000; 9: 181-184.

19. Cascorbi I, Henning S, Brocmoller J, et al. Substantially reduced risk of cancer of the aerodigestive tract in subjects with variant $-463 \mathrm{~A}$ of the myeloperoxidase gene. Cancer Res 2000; 60: 644-649.

20. Chen H, Lum A, Seifried A, Wilkens LR, Le Machand L. Association of the NAD $(\mathrm{P}) \mathrm{H}$ :quinone oxidoreductase $609 \mathrm{C} \rightarrow \mathrm{T}$ polymorphism with a decreased lung cancer risk. Cancer Res 1999; 59: 3.045-3.048.

21. Harth V, Donat S, Ko Y, Abel J, Vetter H, Brunning $T$. NAD (P)H quinone oxidoreductase 1 codon 609 polymorphism and its association to colorectal cancer. Arch Toxicol 2000; 73: 528-531.

22. Smith CA, Harrison DJ. Association between polymorphism in gene for microsomal epoxide hydrolase and susceptibility to emphysema. Lancet 1997; 350: $630-633$.

23. Benhamou S, Reinikainen M, Bouchardy C, Dayer P, Hirvonen A. Association between lung cancer and microsomal epoxide hydrolase genotypes. Cancer Res $1998 ; 58: 5.291-5.293$.

24. Houlston RS. CYPIA1 polymorphisms and lung cancer risk: a meta-analysis. Pharmacogenetics 2000; 10: 105114 .

25. Christensen PM, Gotzsche PC, Brosen K. The sparteine/debrisoquine (CYP2D6) oxidation polymorphism and the risk of lung cancer: a meta-analysis. Eur J Clin 
Pharmacol 1997; 51: 389-393.

26. Rostami-Hodjegan A, Lennard MS, Woods HF, Tucker GT. Meta-analysis of studies of the CYP2D6 polymorphism in relation to lung cancer and Parkinson's disease. Pharmacogenetics 1998; 8: 227-238.

27. Milner BJ, Brown I, Gabra H, Kitchener HC, Parkin DE, Haites NE. A protective role for common p21WAF1/Cip1 polymorphisms in human ovarian cancer. Int J Oncol 1999; 15: 117-119.

28. Houlston RS. Glutathione S-transferase MI status and lung cancer risk: a meta-analysis. Cancer Epidemiol Biomarkers Prev 1999; 8: 675-682.

29. Ozdemir E, Kakehi Y, Nakamura E, et al. HLADRB1*0101 and *0405 as protective alleles in Japanese patients with renal cell carcinoma. Cancer Res 1997; 57: $742-746$.

30. Ichimiya M, Muto M, Hamamoto $\mathrm{Y}$, Ohmura A, Tateno H, Asagami C. Putative linkage between HLA class I polymorphism and the susceptibility to malignant melanoma. Australas J Dermatol 1996; 37 (Supl 1) : S39.

31. Tokumoto H. Analysis of HLA-DRB1-related alleles in Japanese patients with lung cancer-relationship to genetic susceptibility and resistance to lung cancer. J Cancer Res Clin Oncol 1998; 124: 511-516.

32. Dunning AM, Chiano M, Smith NR, et al. Common BRCA1 variants and susceptibility to breast and ovarian cancer in the general population. Hum Mol Genet $1997 ; 6: 285-289$.

José Luis Pérez-Gracia and María Gloria Ruiz-Ilundáin

a Clinical Research Department. Eli Lilly and Company.

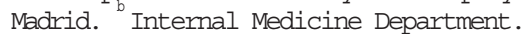
Hospital Universitario 12 de Octubre. Madrid. 